International Journal of Pure and Applied Mathematics

Volume 95 No. 4 2014, 547-552

ISSN: 1311-8080 (printed version); ISSN: 1314-3395 (on-line version)

url: http://www.ijpam.eu

doi: http://dx.doi.org/10.12732/ijpam.v95i4.7

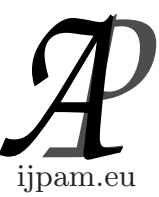

\title{
REFLEXIVITY IN ASSOCIATIVE TRIPLE SYSTEMS
}

\author{
V. Ramaswamy ${ }^{1}$, D. Rajalakshmi ${ }^{2} \S$ \\ Principal, BIET \\ Davangere, Karnataka, 577 004, INDIA \\ ${ }^{2}$ Department of Mathematics \\ Srinivasa Ramanujan Centre \\ SASTRA University, Kumbakonam, Tamil Nadu, 612 001, INDIA
}

\begin{abstract}
In the present paper, we define the notions of regularity, strong regularity and reflexivity in associative triple systems and prove some theorems concerning these notions.
\end{abstract}

AMS Subject Classification: 05B07

Key Words: regularity, strong regularity, reflexivity

\section{Introduction and Preliminaries}

A vector space $T$ over a field $F$ together with a trilinear map $(x, y, z) \rightarrow<x, y, z>$ is called a triple system. A triple system $T$ is said to be an associative triple system if $\langle\langle x, y, z\rangle, u, v\rangle=\langle x,\langle y, z, u\rangle, v\rangle$ $=\langle x, y,\langle z, u, v\rangle\rangle$ for all $x, y, z, u, v \in T$. For example, if $A$ is an associative algebra, then the underlying vector space of $A$ together with the map $(x, y, z) \rightarrow x y z$ is an associative triple system which we denote by $T_{A} . T_{A}$ is called the triple system associated with the algebra $A$. Hence forward, $T$ will denote an associative triple system. We call an element $x$ in $T$ invertible if there exists an element $x_{1} \in T$ such that $\left.\left\langle x, x_{1}, t\right\rangle=\left\langle x_{1}, x, t\right\rangle=<t, x, x_{1}\right\rangle=<$ $t, x_{1}, x>=t$ for all $t \in T . x_{1}$ is called an inverse of $x$. It can be easily seen that an invertible element of a triple system $T$ has a unique inverse. Triple system $T$

Received: April 28, 2014

(C) 2014 Academic Publications, Ltd.

${ }^{\S}$ Correspondence author url: www.acadpubl.eu 
is said to be a division triple system if every non zero element of $T$ is invertible. It can be easily shown that $T$ is a division triple system if and only if the following

condition is satisfied: For $a, b, c \in T(a, b \neq 0)$, the equations $\langle a, b, x\rangle$ $=c,\langle a, x, b\rangle=c$ and $\langle x, a, b\rangle=c$ have solutions. If $A$ is a division algebra, then the triple system $T_{A}$ associated with $A$ is a division triple system.

The odd powers of an element $a \in T$ are defined recursively as follows. $a^{1}$ $=a, a^{[2(n+1)+1]}=\left\langle a^{(2 n+1)}, a, a>\right.$. $a$ is said to be nilpotent if $a^{(2 n+1)}=0$ for some positive integer $n$. For $x \in T$, we define a map $U_{x}: T \rightarrow T$ as $U_{x}(y)=$ $\langle x, y, x\rangle$. $x$ is said to be a zero divisor if $U_{x}$ is not injective.

An element $a \in T$ is said to be regular if there exist an element $x \in T$ such that $a=U_{a}(x)=\langle a, x, a\rangle$. $x$ is referred to as a generalized inverse of $a . a$ is said to be unit regular if there exists an invertible element $x$ such that $a=$ $U_{a}(x)=\langle a, x, a\rangle$. $a$ is said to be strongly regular if there exists an element $x \in T$ such that $a=\langle a, a, x\rangle$. $T$ is said to be strongly regular if every element of $T$ is strongly regular. An element $x$ is said to be a reflexive inverse of $a$ if $a=U_{a}(x)$ and $x=U_{x}(a)$. If $x$ is a reflexive inverse of $a$, then it follows that $a$ is regular with $x$ as a generalized inverse.

\section{Main Results}

The relationship between regularity and strong regularity is given by the following.

Theorem 2.1. For each element $a$ of a strongly regular triple system $T$, there corresponds an element $x$ such that $\langle a, a, x\rangle=\langle a, x, a\rangle=\langle x, a, a\rangle$ $=a$. Thus every element of a strongly regular triple system $T$ is regular.

Proof. We will first prove that a strongly regular triple system $T$ cannot contain any nonzero nilpotent element. Suppose $s \in T$ and $s$ is nilpotent. Then $s^{(2 p+1)}=0$ for some positive integer $p$. Since $T$ is strongly regular, there exists $t \in T$ such that $s=\langle s, s, t\rangle$. Now $s=\langle s, s, t\rangle=\langle s,\langle s, s, t\rangle, t\rangle=$ $<s^{3}, t, t>$ (by associativity). We will now prove that $s=\left\langle s^{(2 n+1)}, t^{(2 n-1)}, t>\right.$ by induction on $n$. The result is true when $n=1$. Now assume that $s=$ $<s^{(2 n+1)}, t^{(2 n-1)}, t>$. Then

$$
\begin{aligned}
s & =<<s^{(2 n-1)}, s, s>, t^{(2 n-1)}, t> \\
& =<<s^{(2 n-1)}, s,<s, s, t>>, t^{(2 n-1)}, t>\quad(\text { note that } s=<s, s, t>) \\
& =<s^{(2 n+1)}, s, t^{(2 n+1)}>(\text { by associativity) }
\end{aligned}
$$




$$
\begin{aligned}
& =<s^{(2 n+1)},<s, s, t>, t^{(2 n+1)}>\text { since } s=<s, s, t> \\
& =<s^{(2 n+3)}, t^{(2 n+1)}, t>\text { (by associativity) }
\end{aligned}
$$

We have thus proved that $s=<s^{(2 n+1)}, t^{(2 n-1)}, t>$ for all positive integers $n$. Since $s^{(2 p+1)}=0$, we conclude that $s=0$. We will now come to the proof of the theorem. Since $T$ is strongly regular, so is $a$. Hence there exists an element $x$ such that $a=\langle a, a, x\rangle$. Using associativity of $T$, we can easily prove that $(a-\langle a, x, a\rangle)=0$. Therefore $a-\langle a, x, a\rangle$ is a nilpotent element of the strongly regular triple system $T$. By what we have proved above, we conclude that $a-\langle a, x, a\rangle=0$ showing that $a=\langle a, x, a\rangle$. Similarly, we can prove that $a=\langle x, a, a\rangle$.

Theorem 2.2. For a nonzero regular element $a$ of a triple system $T$, the following statements are equivalent:

(i) $a$ has a unique generalized inverse.

(ii) $a$ is not a zero divisor.

(iii) There exist an element $x \in T$ such that $\langle x, a, t\rangle=\langle t, a, x\rangle=t$ for all $t$.

Proof. (i) $\Rightarrow$ (ii) Let $x$ be the unique generalized inverse of $a$. Then $a=$ $U_{a}(x)=\langle a, x, a\rangle$. Let $t \in T$ such that $U_{a}(t)=0$. Now $U_{a}(x+t)=$ $\langle a, x+t, a\rangle=\langle a, x, a\rangle+\langle a, t, a\rangle=a$ since $\langle a, t, a\rangle=0$. Hence $x+t$ is also a generalized inverse of $a$. By the uniqueness of $x$, we conclude that $x+t=x$ so that $t=0 . U_{a}$ is thus injective proving that $a$ is not a zero divisor.

(ii) $\Rightarrow$ (iii)

Since $a$ is regular, we can find an element $x \in T$ such that $a=U_{a}(x)=$ $\langle a, x, a\rangle$. Let $t \in T$ be arbitrary. Then Since $a$ is not a zero divisor, $U_{a}$ is injective so that $t-\langle x, a, t\rangle=0$ proving that $t=\langle x, a, t\rangle$. Similarly, we can prove that $t=\langle t, a, x\rangle$ for all $t \in T$.

(iii) $\Rightarrow$ (i)

Since $a$ is regular, $a$ has a generalized inverse say $t$ so that $a=U_{a}(t)=$ $<a, t, a>$. By (iii), there exists an element $x \in T$ such that $\langle x, a, t\rangle=\langle t, a, x\rangle=t$. Now $\langle x, a, x\rangle=\langle x,\langle a, t, a\rangle, x\rangle=$ $<<x, a, t>, a, x\rangle=<t, a, x\rangle=t$. This proves that $t$ is unique.

Theorem 2.3. For a non zero element $a$ of a strongly regular triple system $T$, the following statements are equivalent. 
(i) a has a unique generalized inverse.

(ii) $a$ is not a zero divisor.

(iii) a is invertible.

Proof. (i) $\Rightarrow$ (ii) Since $T$ is strongly regular, it follows from theorem that $a$ is regular so that by theorem, $a$ is not a zero divisor.

(ii) $\Rightarrow$ (iii) Since $T$ is strongly regular, it follows from the theorem that there exists an element $x$ such that $a=\langle a, a, x\rangle=\langle a, x, a\rangle=\langle x, a, a\rangle$. Also by the theorem, $\langle x, a, t\rangle=\langle t, a, x\rangle=t$ for all $t \in T$. Now, $U_{a}(t-<a, x, t>)=\langle a, t-<a, x, t>, a\rangle=\langle a, t, a\rangle-$ $<a,\langle a, x, t>, a\rangle=\langle a, t, a\rangle-<<a, a, x>, t, a\rangle=\langle a, t, a\rangle-<$ $a, t, a>=0$. Since $a$ is not a zero divisor, $U_{a}$ is injective so that $t=\langle a, x, t\rangle$. Similarly using $a=\langle x, a, a\rangle$, we can prove that $t=\langle t, x, a\rangle$. $x$ is thus the inverse of $a$.

(iii) $\Rightarrow$ (i) Since by Theorem, $a$ is regular, (iii) $\Rightarrow($ i) follows from Theorem 2.2.

Theorem 2.4. $T$ is a division triple system if and only if every non zero element of $T$ is strongly regular with a unique generalized inverse.

Proof. If $T$ is a division triple system, then every nonzero element $a \in T$ has an inverse say $a^{(-1)}$. Hence $\left\langle a, a, a^{(-1)}\right\rangle=a$ which proves that $a$ is strongly regular. Thus $T$ is a strongly regular triple system in which a is invertible so that by Theorem 2.3, a has a unique generalized inverse. Conversely, if every non zero element of $T$ is strongly regular with a unique generalized inverse, then $T$ is a strongly regular triple system so that by Theorem 2.3, every non zero element of $T$ is invertible. Hence $T$ is a division triple system.

Lemma 2.5. If $a$ is a regular element of $T$ with a generalized inverse $x$, then $\langle x, a, x\rangle$ is a reflexive inverse of $a$.

Proof. $x$ is a generalized inverse of $a$ means $a=U_{a}(x)=\langle a, x, a\rangle$. If $y$ $=\langle x, a, x\rangle$, then $U_{a}(y)=\langle a, y, a\rangle=\langle a,\langle x, a, x\rangle, a\rangle=\langle<a, x, a\rangle$ $, x, a\rangle=\langle a, x, a\rangle=a$. Again $U_{y}(a)=<y, a, y>=<<x, a, x>, a,<$ $x, a, x \quad>>\quad<\quad x,<\quad a, x, a \quad>,<\quad x, a, x \quad>>=$ $\langle x, a,\langle x, a, x\rangle\rangle=\langle x,\langle a, x, a\rangle, x\rangle=\langle x, a, x\rangle=y$. This shows that $y$ is a reflexive inverse of $a$. We call an element $x \in T$ a strong reflexive inverse of $a$ if $\langle a, a, x\rangle=\langle a, x, a\rangle=\langle x, a, a\rangle=a$ and $\langle x, x, a\rangle=\langle x, a, x\rangle$ $=\langle a, x, x\rangle=x$. 
Theorem 2.6. If $a$ is an element of $T$ with a unique reflexive inverse $x$, then $\langle a, a, x\rangle=\langle x, a, a\rangle$ and $\langle a, x, x\rangle=\langle x, x, a\rangle$.

Proof. Since $x$ is a reflexive inverse of $a$, we have $U_{a}(x)=a$ and $U_{x}(a)=$ $x$. For $y \in T$, we have $U_{a}(x+y-\langle x, a, y\rangle)=\langle a, x+y-\langle x, a, y\rangle, a\rangle$ $=\langle a, x, a\rangle+\langle a, y, a\rangle-\langle a,\langle x, a, y\rangle, a\rangle=U_{a}(x)+U_{a}(y)-$ $<<a, x, a\rangle, y, a\rangle=U_{a}(x)+U_{a}(y)-U_{a}(y)$ since $\langle a, x, a\rangle=U_{a}(x)=a$ $=U_{a}(x)=a$ Also $U_{a}(x+y-\langle y, a, x\rangle)=\langle a, x+y-\langle y, a, x\rangle, a\rangle$ $=\langle a, x, a\rangle+\langle a, y, a\rangle-\langle a,<y, a, x\rangle, a\rangle=U_{a}(x)+U_{a}(y)-$ $<a, y,<a, x, a>>=U_{a}(x)+U_{a}(y)-U_{a}(y)=U_{a}(x)=a$. Thus $x+y-$ $\langle x, a, y\rangle$ and $x+y-\langle y, a, x\rangle$ are both generalized inverses of $a$. From the Lemma, it follows that $\langle x+y-\langle x, a, y\rangle, a, x+y-\langle x, a, y\rangle\rangle$ and $\langle x+y-\langle y, a, x\rangle, a, x+y-\langle y, a, x\rangle\rangle$ are both reflexive inverses of $a$. Since $x$ is the unique reflexive inverse of $a$, we have $x=<x+y-$ $\langle x, a, y\rangle, a, x+y-\langle x, a, y\rangle\rangle$. Using associativity of $T,\langle x, a, x\rangle$ $=x$ and $\langle a, x, a\rangle=a$ and simplifying, we obtain $x=x+\langle y, a, x\rangle-$ $<<x, a, y\rangle, a, x\rangle$ so that $\langle y, a, x\rangle=\langle<x, a, y\rangle, a, x\rangle$ for all $y \in T$. Similarly, using the fact that $\langle x+y-\langle y, a, x\rangle, a, x+y-\langle y, a, x\rangle\rangle$ is a reflexive inverse of $a$, we can prove that $\langle x, a, y\rangle=\langle x, a,\langle y, a, x\rangle\rangle$ for all $y \in T$. Thus for all $y \in T$,

$$
<x, a, y>=<y, a, x>
$$

Taking $y=a$ in 2.1, we obtain $\langle x, a, a\rangle=\langle a, a, x\rangle$. Again, taking $y$ $=\langle x, x, a\rangle$ in 2.1, we obtain $\langle x, a,\langle x, x, a\rangle\rangle=\langle<x, x, a\rangle, a, x\rangle$ i.e $<<x, a, x\rangle, x, a\rangle=\langle<x, x, a\rangle, a, x\rangle$. Since $\langle x, a, x\rangle=x$, we have

$$
<x, x, a>=<<x, x, a>, a, x>=<x,\langle x, a, a>, x>
$$

Taking $y=\langle a, x, x\rangle$ in 2.1, we obtain $\langle x, a,<a, x, x\rangle\rangle=$ $<<a, x, x\rangle, a, x\rangle$ so that $\langle x,<a, a, x\rangle, x\rangle=\langle a, x,<x, a, x\rangle\rangle$. i.e $\langle x,\langle a, a, x\rangle, x\rangle=\langle a, x, x\rangle$ since $\langle x, a, x\rangle=x$. Since we have already proved that $\langle a, a, x\rangle=\langle x, a, a\rangle$, this implies that

$$
<x,<x, a, a>, x>=<a, x, x>
$$

From 2.2 and 2.3 we obtain $\langle x, x, a\rangle=\langle a, x, x\rangle$. This completes the proof of the theorem.

Theorem 2.7. Let $a$ be an element of $T$ with a reflexive inverse $x$ such that $\langle x, x, a\rangle=x$. Further, let $x$ be the unique generalized inverse of $a$. Then $a$ is invertible. 
Proof. Since $x$ is a reflexive inverse of $a$, we have $a=U_{a}(x)=\langle a, x, a\rangle$ and $x=U_{x}(a)=\langle x, a, x\rangle$. Since $x$ is the unique generalized inverse of $a$, $x$ is also the unique reflexive inverse of $a$. Hence it follows from Theorem 2.6 that $\langle a, a, x\rangle=x, a, a\rangle$ and $\langle a, x, x\rangle=\langle x, x, a\rangle$. This together with the hypothesis gives $\langle a, x, x\rangle=\langle x, x, a\rangle=\langle x, a, x\rangle=x$. Now for any $t \in T$, we have $\langle a, x, t\rangle=\langle<a, x, a\rangle, x, t\rangle=\langle a,\langle x, a, x\rangle, t\rangle=$ $\langle a,\langle x, x, a\rangle, t\rangle=\langle\langle a, x, x\rangle, a, t\rangle=\langle x, a, t\rangle$. Similarly, we can prove that $\langle t, a, x\rangle=\langle t, x, a\rangle$ for all $t \in T$. Now by Theorem $2.2,\langle x, a, t\rangle$ $=\langle t, a, x\rangle=t$ for all $t \in T$ so that $\langle a, x, t\rangle=\langle x, a, t\rangle=\langle t, a, x\rangle=$ $<t, x, a\rangle=t$ for all $t \in T$ proving that $a$ is invertible with inverse $x$.

Theorem 2.8. Let $a$ be an element of $T$ possessing a reflexive inverse. Furthermore, let $\langle a, x, t\rangle=\langle x, a, t\rangle$ and $\langle t, a, x\rangle=\langle t, x, a\rangle$ for all $t \in T$ and for all generalized inverses $x$ of $a$. Then $a$ has a unique reflexive inverse.

Proof. Let $y$ and $z$ both be reflexive inverses of $a$. Then $a=\langle a, y, a\rangle=$ $\langle a, z, a\rangle, y=\langle y, a, y\rangle$ and $z=\langle z, a, z\rangle$. Now $\langle a, y, t\rangle=\langle y, a, t\rangle$ (by hypothesis) $=\langle y,\langle a, z, a>, t>=\langle<y, a, z>, a, t>=\langle<a, y, z\rangle$ $, a, t>$ (by hypothesis since $\mathrm{y}$ is a generalized inverse) $=\langle a, y,<z, a, t>\rangle$ $=\langle a, y,\langle a, z, t\rangle\rangle$ (again by hypothesis since $\mathrm{z}$ is a generalized inverse) $=$ $<<a, y, a\rangle, z, t\rangle=\langle a, z, t\rangle$. We have thus proved that $\langle a, y, t\rangle=$ $<y, a, t\rangle=\langle a, z, t\rangle=\langle z, a, t\rangle$ for all $t \in T$. Similarly, we can prove that $\langle t, a, y\rangle=\langle t, y, a\rangle=\langle t, a, z\rangle=\langle t, z, a\rangle$ for all $t \in T$. Now $y=$ $\langle y, a, y\rangle=\langle z, a, y\rangle$ (since by above, $\langle y, a, t\rangle=\langle z, a, t\rangle$ for all $t \in T$ ) $=\langle z, a, z\rangle$ (since $\langle t, a, y\rangle=\langle t, a, z\rangle$ for all $t \in T)=z$. This proves that reflexive inverse of $a$ is unique.

\section{References}

[1] . Meyberg K., Lectures on Algebra and Triple Systems, Lecture Notes, The University of Virginia, Charolottesville.

[2] .V. Ramaswamy, Strong regularity in associative triple systems, Acta Ciencia Indica, XIX, No. 4 (1993), 193. 